ICONE19-43913

\title{
VISUALIZATION OF TWO-PHASE INTERFACIAL STRUCTURES IN NARROW CHANNEL
}

\author{
Daisuke Ito \\ ETH Zürich, \\ ML K 14 Sonneggstrasse 3, \\ Zurich, Switzerland \\ Phone:+41 446328784 \\ ito@lke.mavt.ethz.ch
}

\author{
Horst-Michael Prasser \\ ETH Zürich, \\ ML K 13 Sonneggstrasse 3, \\ Zurich, Switzerland \\ Phone:+41 446326025 \\ prasser@lke.mavt.ethz.ch
}

\author{
Masanori Aritomi \\ Tokyo Institute of Technology \\ 2-12-1 Ohokayama, Meguro, \\ Tokyo, Japan \\ Phone: +81 357343063 \\ maritomi@nr.titech.ac.jp
}

Keywords: two-phase flow, narrow channel, electrical conductance, gas-liquid interfacial structure.

\begin{abstract}
The gas-liquid interfacial structure of two-phase flow in narrow channel was measured by a novel measurement method using electrical conductance. This method consists of two different conductance measurements. One measure the conductance between neighbor electrodes on the sensor, and the liquid film thickness on the channel wall can be estimated from the measured conductance. Another measures the conductance between the opposing electrodes of two sensors which are installed on the walls of narrow rectangular channel. This conductance is related directly to void fraction in the narrow gap. These two measurements are performed simultaneously. Thus, two-dimensional distributions of both liquid film thickness and void fraction can be measured by using the liquid film sensors with numerous electrodes. The gas-liquid interfaces were reconstructed by estimating the thickness of the gaseous phase in the gap. From the reconstructed data, the void fraction and interfacial area concentration were obtained.
\end{abstract}

\section{INTRODUCTION}

The fluid passage in the nuclear reactor core and the heat exchanger is relatively small. To clarify the two-phase flow in the narrow channel, many interesting studies have been carried out. Especially, a triangle dense-lattice rod bundle has been adopted as a fuel rod configuration in high conversion boiling water reactor (Iwamura et al. 2006, Uchikawa et al. 2007, Fukaya et al. 2009), which has been developing as next-generation light water reactor. It has a narrow gap of about $1 \mathrm{~mm}$ in the bundle flow channel. Therefore, two-phase flow analysis for the dense-lattice bundle or narrow channel is needed, and the acquisition of experimental data and the modeling of the flow have been done in the past several years. Tamai et al. (2006) evaluated the effect of the gap width and the power profile from the pressure drop measured in dense-lattice 37 rod bundles. Sadatomi et al. (2007) studied the void fraction in a double subchannel with dense-lattice array and the data was compared with some correlations and subchannel codes. Furthermore, their group estimated the wall and the interfacial friction forces from the measured void fraction and pressure drop using the same subchannel (Kawahara et al. 2008). However, the advanced measurement techniques of the spatio-temporal distribution of the flow parameters such as void fraction and velocity are required for the high accurate analysis of the flow.

For the void fraction distribution measurement, the radiation methods have been developed to evaluate the flow in the bundle. Kureta (2007) developed a neutron radiography method for three-dimensional tomographic imaging of two-phase flow. The three-dimensional flow structures in the dense-lattice rod bundle were visualized. Although the spatial void fraction distribution can be obtained by the radiation method, it is difficult to measure the time variation with high temporal resolution. In addition, there are some limitations of the uses. Thus, the authors focused on two-phase flow measurement using electrical conductance. Wire-mesh sensor (WMS), which uses the difference of electrical conductance between gas and liquid phases, has received attention as cross-sectional void fraction distribution measurement method (Prasser et al. 1998). For the application of WMS to the flow in the rod bundle, a lot of electrode wires have to be installed over the cross section, and the intrusive effect to the flow becomes larger. Therefore, a novel void fraction measurement method with the electrodes on the wall of the flow channel was developed for narrow two-phase flow (Ito et al. 2010). The wire electrodes were fixed on the opposing walls of the narrow channel and the conductance in the narrow gap was measured. As a result, three-dimensional void fraction distributions can be obtained. On the other hand, a liquid film sensor based on the electrical conductance measurements was developed (Damsohn and Prasser, 2009). This can estimate the liquid film thickness distribution on the Copyright (C) 2011 by JSME 


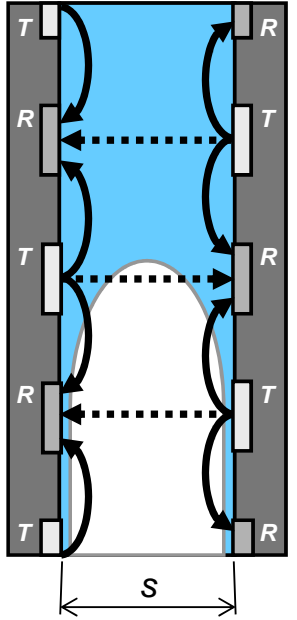

Electrical conductance caused by liquid film thickness

Electrical conductance caused by void fraction

$T:$ Transmitter electrode $R$ : Receiver electrode

Fig. 1 Schematics of simultaneous measurement of

liquid film thickness and void fraction

wall in a flow channel. In addition, this sensor was applied to the annular flow measurement in the double subchannel of a square-lattice bundle. The liquid film behavior in the rod bundle was well visualized (Damsohn and Prasser, 2010).

In this study, the simultaneous measurements of the void fraction and the liquid film thickness in a narrow channel with a gap width of $1.5 \mathrm{~mm}$ are carried out by using two liquid film sensors developed by Damsohn and Prasser (2009). The interfacial structure is visualized by reconstructing the gas-liquid interfaces from the measured void fraction and liquid film thickness. Using the reconstructed data, the void fraction is recalculated and interfacial area concentration is estimated.

\section{MEASUREMENT TECHNIQUE}

A pair of the liquid film sensors is used to measure the two-dimensional distribution of both liquid film thickness and void fraction in the narrow channel, simultaneously. The principle of this measurement is represented in Fig. 1. The liquid film thickness is estimated by measuring the electrical conductance between neighboring electrodes on the same sensor. On the other hand, the conductance between the opposing walls can be converted into the void fraction. The transmitters of two sensors are excited and the electrical conductance is received by all receiver electrodes. As a result, the distributions of liquid film thickness on both walls and void fraction in narrow gap are obtained.

The applied liquid film sensor is shown in Fig. 2. The sensing surface of this sensor consists of the transmitter, the receiver and the ground electrodes. These are the circular electrodes. The transmitter and receiver electrodes have a diameter of $0.5 \mathrm{~mm}$ and the ground electrode has $0.9 \mathrm{~mm}$ in diameter. The distance between each electrode is $1 \mathrm{~mm}$. Therefore, this geometry allows the spatial resolution of $2 \mathrm{~mm}$ for the estimation of the liquid film thickness.

In order to apply this sensor to the liquid film measurements, the calibration for the film thickness is performed to make a polynomial curve, which is used to estimate the liquid film thickness from the measured conductance. The cylinders with different groove depth from

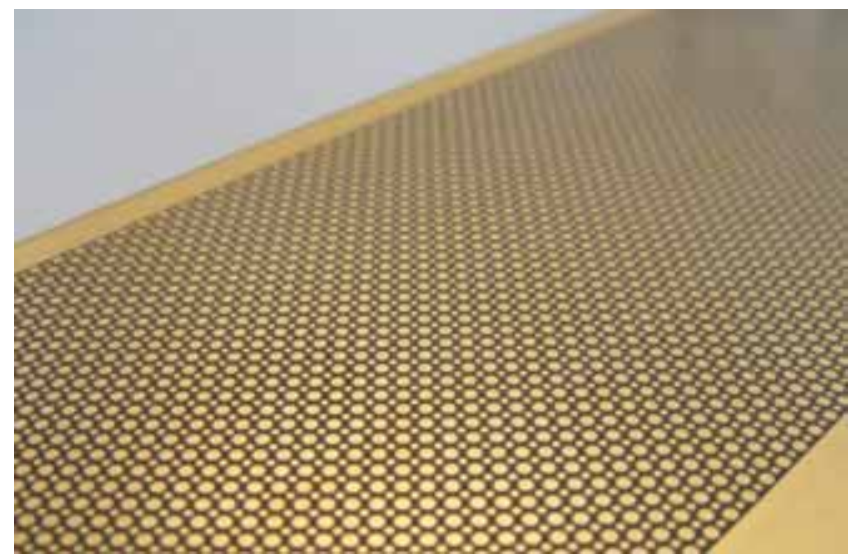

Fig. 2 Sensing surface of applied film sensor

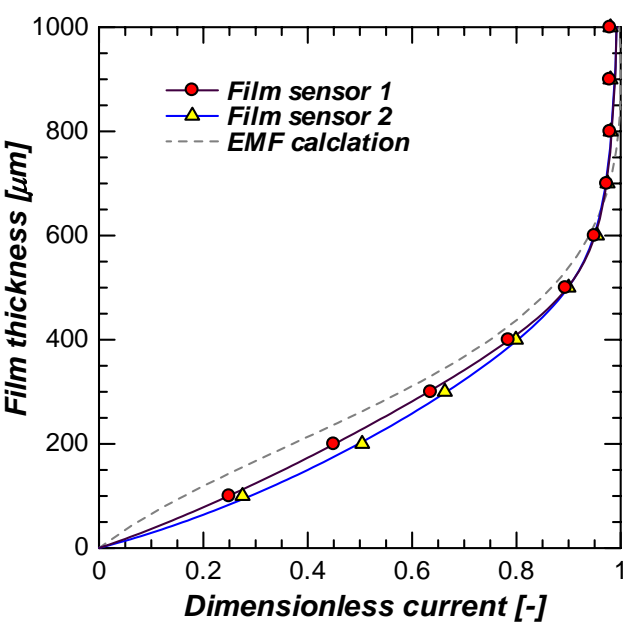

Fig. 3 Calibration result for liquid film thickness

$100 \mu \mathrm{m}$ to $1000 \mu \mathrm{m}$ are rolled over the film sensor in water. Then, the electrical conductance corresponding to each groove depth is obtained for all measurement points, individually. The calibration results of two film sensors are shown in Fig. 3. The dimensionless current is a ratio of the measured electrical conductance to the saturated conductance value. The circular and triangular points are the measured values for each sensor. The solid lines are the fourth order polynomial curve estimated from the measured results. It is seen that the sensor characteristics are different for two sensors. In addition, the result of electromagnetic field (EMF) analysis is also represented in the same figure. Although there is a little difference between the calculation and the measurement, the tendency of the curve is in good agreement.

The liquid film thickness is calculated from the measured conductance by using the coefficients of a polynomial curve with fourth order, as follows;

$$
\delta=\frac{a_{1} X^{4}+a_{2} X^{3}+a_{3} X^{2}+a_{4} X+a_{5}}{\left(X-b_{1}\right)\left(X-b_{2}\right)}
$$

where the coefficients $a_{1} \sim a_{5}$ are determined by the calibration results. $b_{1}$ and $b_{2}$ are the poles of the calibration function. Here, $X$ is defined as a ratio of the conductance as follows.

$$
X=\frac{\sigma_{\text {wall }}}{\sigma_{L}}
$$

where $\sigma_{\text {wall }}$ is the measured conductance on the wall and $\sigma_{L}$ is 


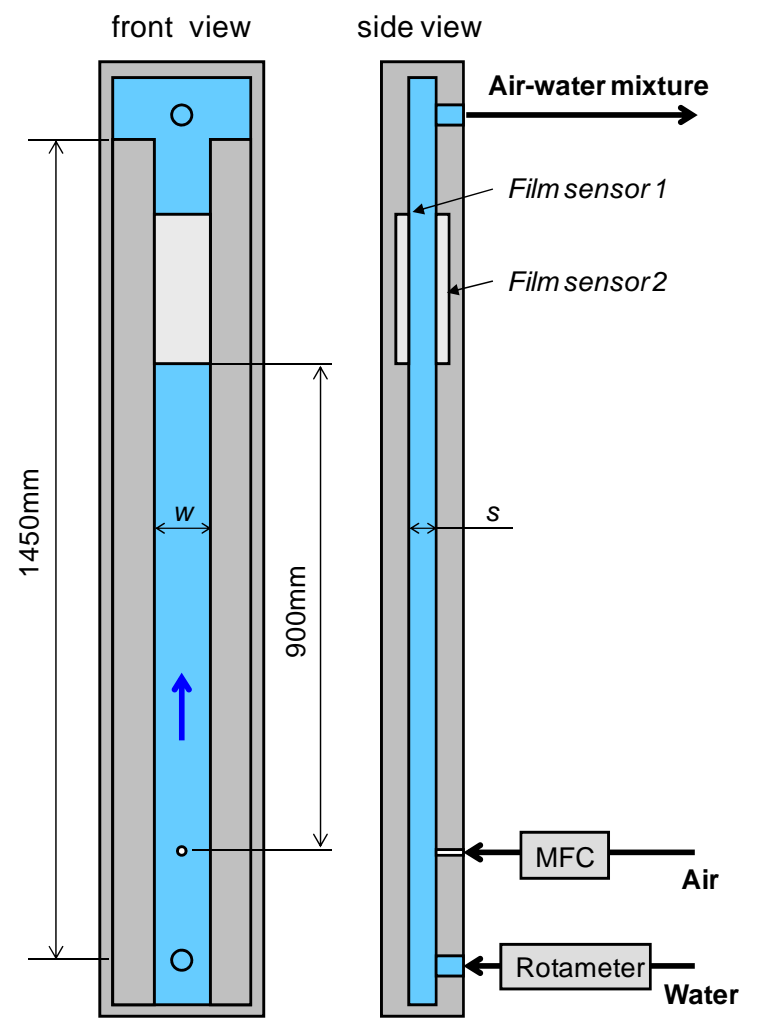

Fig. 4 Experimental set-up

Table 1 Experimental conditions

\begin{tabular}{cc}
\hline Channel cross-section & $w=32 \mathrm{~mm}$, \\
Working fluids & $s=1.5 \mathrm{~mm}$ \\
Superficial liquid velocity & Air and water \\
Superficial gas velocity & $J_{L}=0.17 \mathrm{~m} / \mathrm{s}$ \\
& $J_{G}=0.024 \sim 1.8 \mathrm{~m} / \mathrm{s}$ \\
Flow regimes & Bubbly flow, \\
& slug flow \\
& and churn flow \\
\hline
\end{tabular}

the conductance at the saturated condition of water.

In the void fraction estimation, the linear approximation between the void fraction and the measured conductance is applied and the void fraction is calculated by the following equation.

$$
\alpha_{c}=1-\frac{\sigma_{g a p}}{\sigma_{L}}
$$

where $\sigma_{\text {gap }}$ is the measured conductance between the opposing electrodes. Although the film thicknesses are measured on two walls, the void fractions are estimated by the conductance from both walls. As a result, the measurement points of the void fraction are twice as many as that of the liquid film thickness. Therefore, the interpolation method is applied to uniform the points of both measurements.

In this measuring system for the acquisition of the electrical conductance which is used to estimate the liquid film thickness and void fraction, the maximum sampling rate is $5,000 \mathrm{~Hz}$ for the use of 32 transmitters and 128 receivers. Therefore, the conductance is measured at $32 \times 128(4,096)$ points. Half of them are used for the liquid film measurement and another half is for the void fraction distribution.

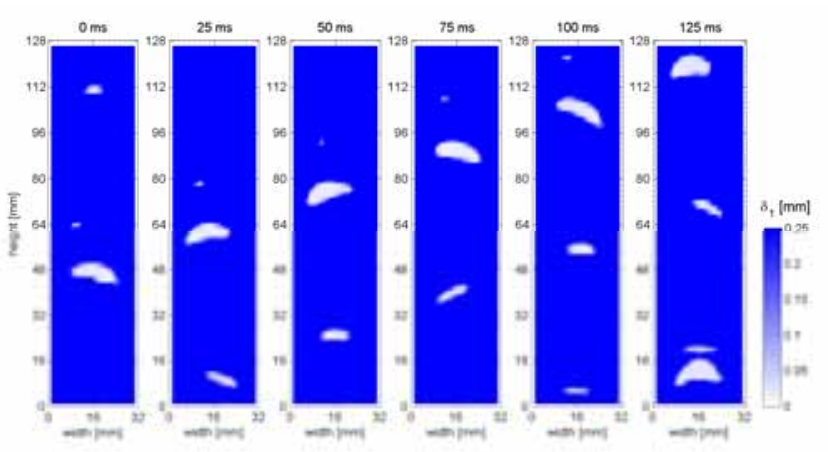

(a) Liquid film thickness on film sensor 1

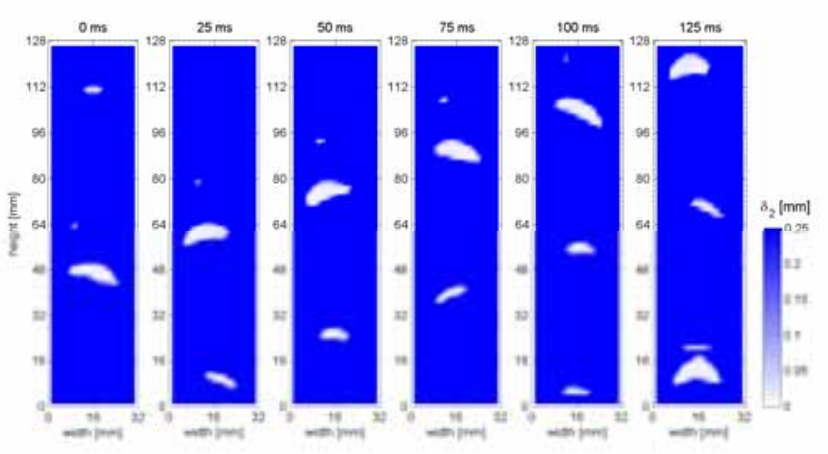

(b) Liquid film thickness on film sensor 2

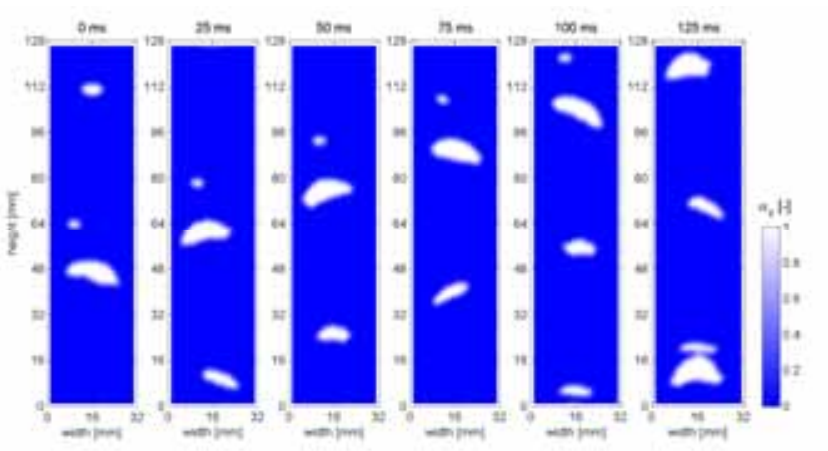

(c) Void fraction between narrow gap

Fig. 5 Instantaneous distributions of liquid film thickness and void fraction in bubbly flow

\section{EXPERIMENTAL SET-UP}

A schematic diagram of the experimental set-up is shown in Fig. 4, and the specifications of the experimental conditions are shown in Table 1. The flow channel has a narrow gap $s$ and a width $w$. In this study, $s$ is $1.5 \mathrm{~mm}$ and $w$ is $32 \mathrm{~mm}$. The working fluids are air and water at room temperature and atmospheric pressure. Water is supplied from the bottom part of the channel. A rotameter is used to monitor the liquid flow rate in the measurement. Air is injected from an orifice placed on a wall of the flow channel. The diameter of the orifice is $1 \mathrm{~mm}$ and the location is the centre of channel width. The air flow rate is controlled by a mass flow controller (MFC). Air-water mixture passes through the measurement section, and then it flows out from the upper part of the channel. The total length of the flow channel is $1450 \mathrm{~mm}$, and two film sensors are installed on both walls at $900 \mathrm{~mm}$ from the air inlet. A film sensor Copyright (C) 2011 by JSME 


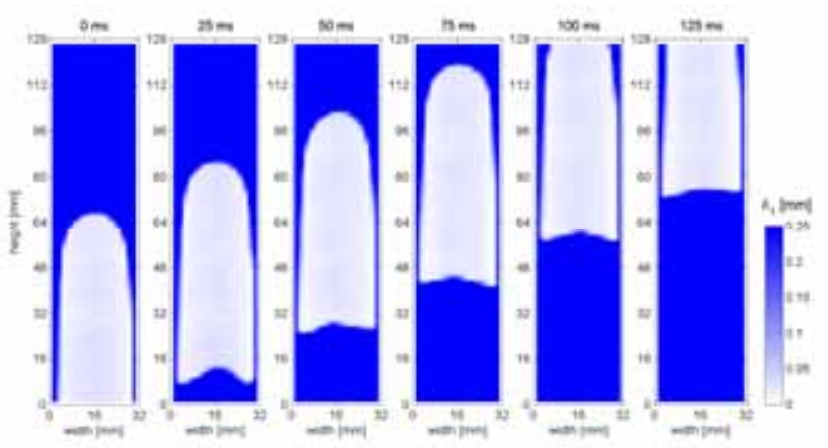

(a) Liquid film thickness on Film sensor 1

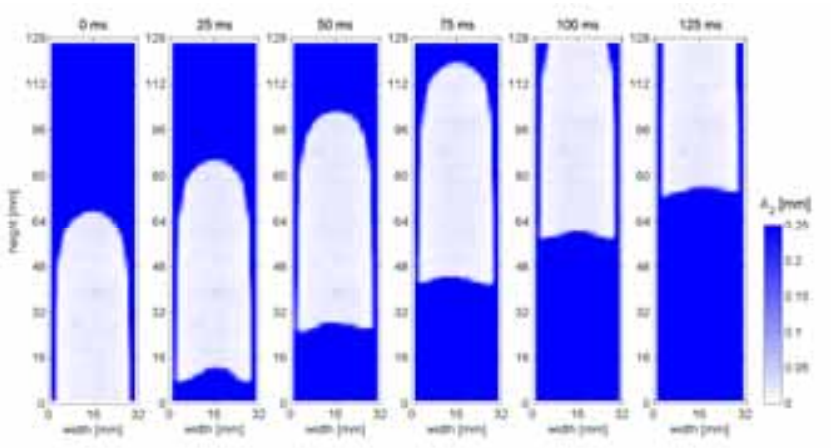

(b) Liquid film thickness on Film sensor 2

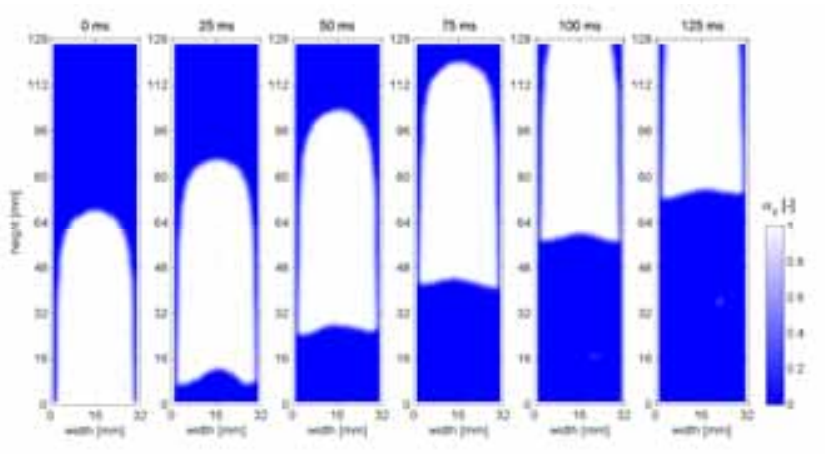

(c) Void fraction in narrow gap

Fig. 6 Instantaneous distributions of liquid film thickness and void fraction in slug flow

installed on the front wall is "Film sensor 1" and another one is "Film sensor 2". In the measuring section, the bubbly, slug and churn flows are formed by varying the flow rate of the air (superficial gas velocity).

\section{RESULTS}

4.1 Instantaneous distributions of liquid film thickness and void fraction

The two-dimensional distributions of the liquid film thickness and the void fraction estimated from the electrical conductance are shown in Figs. 5 7. The values of the film thickness and the void fraction are represented by the color map. Fig. 5 shows the result of the bubbly flow. The bubbles in the measurement area can be seen not only in the void fraction distribution but also in the liquid film thickness one. In addition, the liquid film of these bubbles has very thin

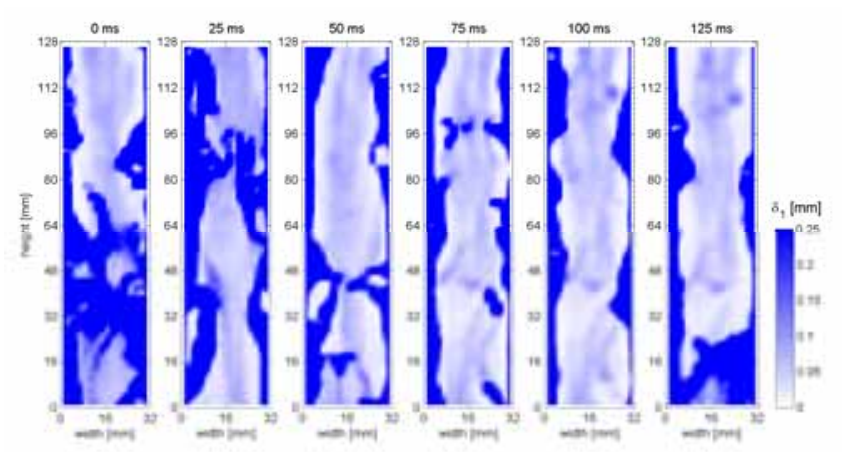

(a) Liquid film thickness on Film sensor 1

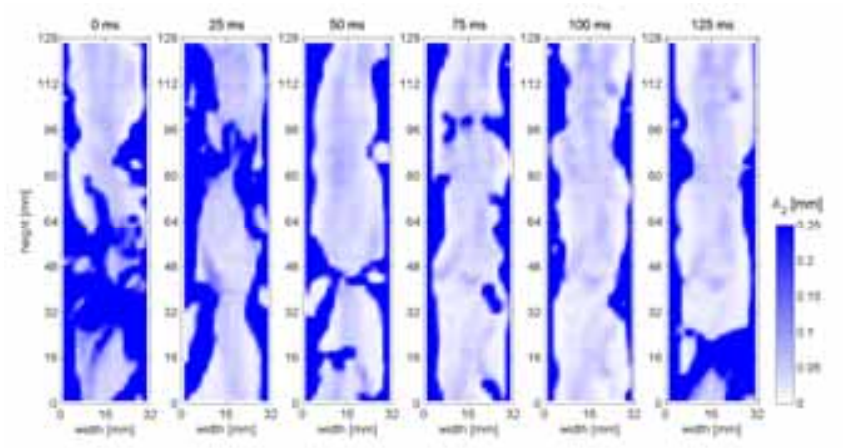

(b) Liquid film thickness on Film sensor 2

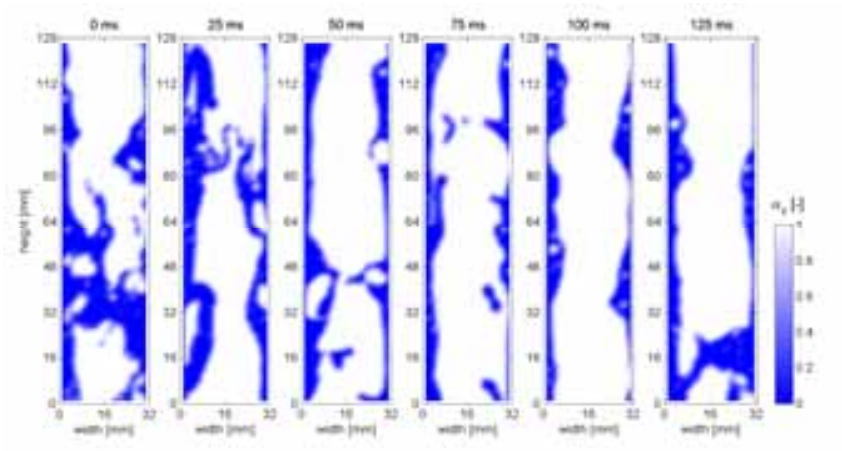

(c) Void fraction in narrow gap

Fig. 7 Instantaneous distributions of liquid film thickness and void fraction in churn flow

thickness and it is less than $50 \mu \mathrm{m}$. In the slug flow, the shape of a Taylor bubble is estimated as shown in Fig. 6. It is found that the liquid film thickness between the Taylor bubble and the wall is also small. It has the film thickness of about $20 \sim 30 \mu \mathrm{m}$ at the centre of the bubble. On the other hand, the gaseous structure becomes complex and the liquid film behaviors are significant in the churn flow in Fig. 7. The film thickness distribution on the gas phase can be visualized by the simultaneous measurement of the liquid film and void fraction.

When the bubble larger than the measurement volume comes to a measurement point, the electrical conductance should be almost zero. Then, although the void fraction estimated from the electrical conductance is $100 \%$ at the point, the actual void fraction differs from the measured value because of the existence of the liquid film layer. For example, 

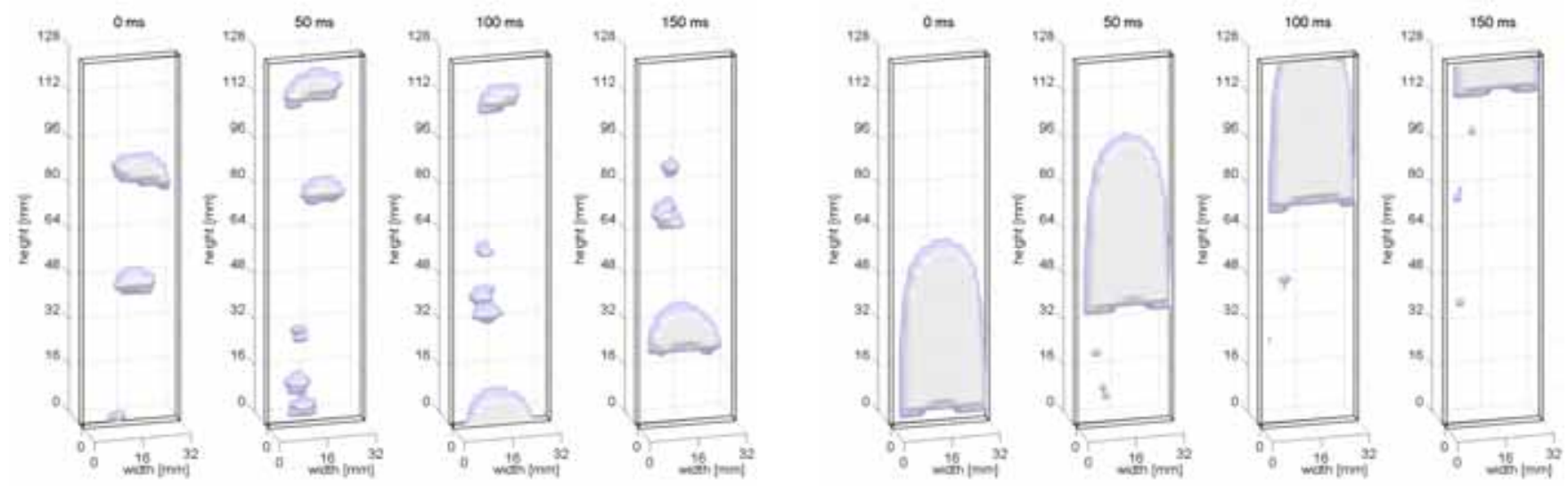

(a) Bubbly flow

(b) Small Taylor bubble
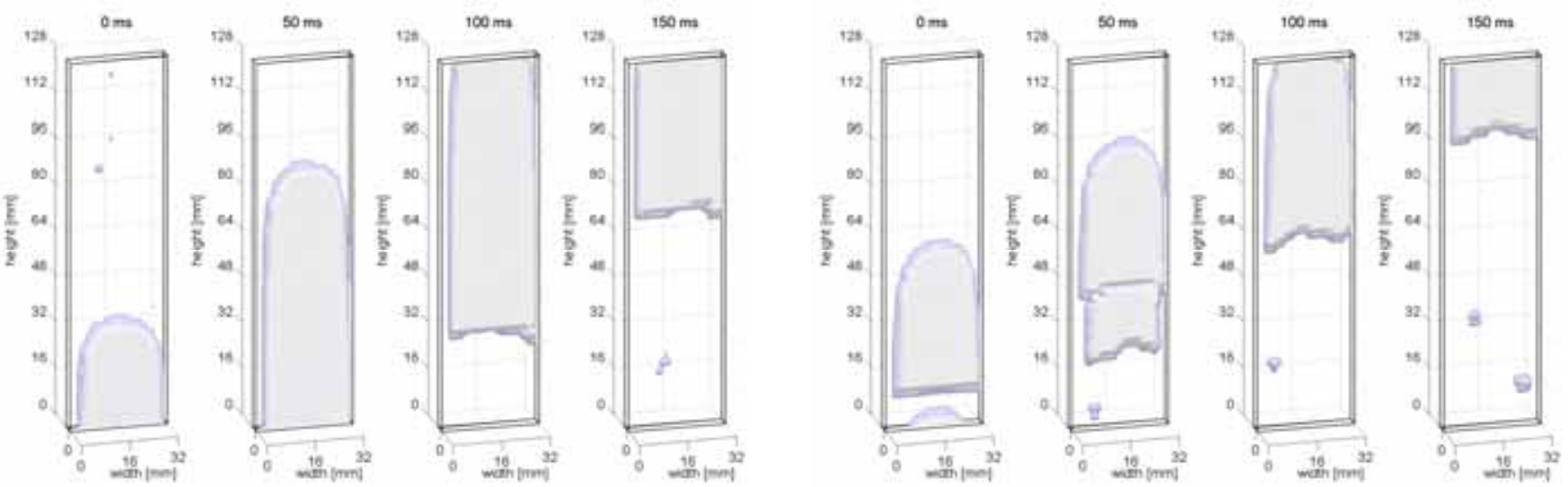

(c) Large Taylor bubble

(d) Coalescence of Taylor bubbles
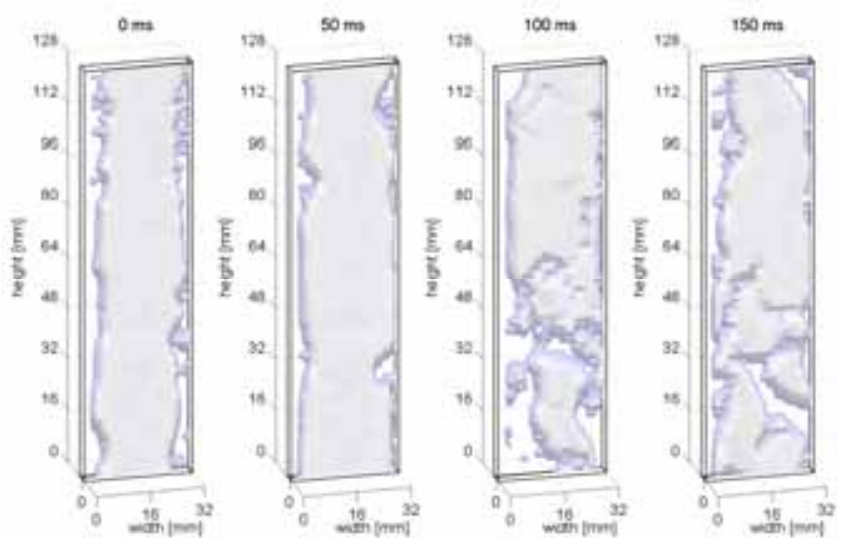

(e) Churn flow
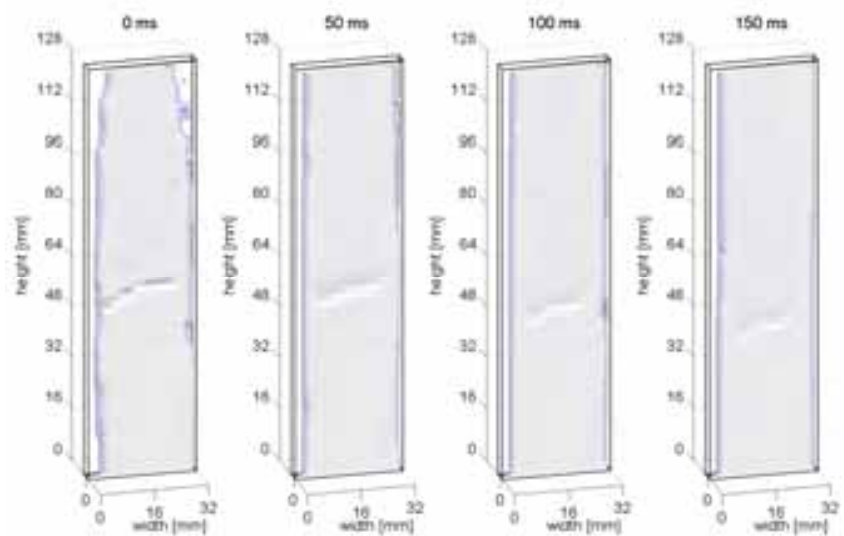

(f) Churn flow with a spot of liquid film

Fig. 8 Reconstructed images of gas-liquid interfaces

when the liquid film thicknesses are $30 \mu \mathrm{m}$ on the both walls and the local void fraction is $100 \%$ at a given point, the actual void fraction in the narrow gap is calculated as $\left(s-\delta_{1}-\delta_{2}\right) / s=$ $(1500-2 \times 30) / 1500=96 \%$. So there is a difference of $4 \%$. This difference increases as the film thickness is larger. Thus, more accurate void fraction estimation is available by considering the liquid film thickness.

\subsection{Reconstruction of gas-liquid interfaces}

The gas-liquid interface is estimated from the measured data of both liquid film thickness and void fraction to visualize the flow structure and to estimate the interfacial structure. Here, the thickness of the gaseous phase (bubble thickness) in the gap direction is defined at all measurement points in both wall sides by the following equations. 

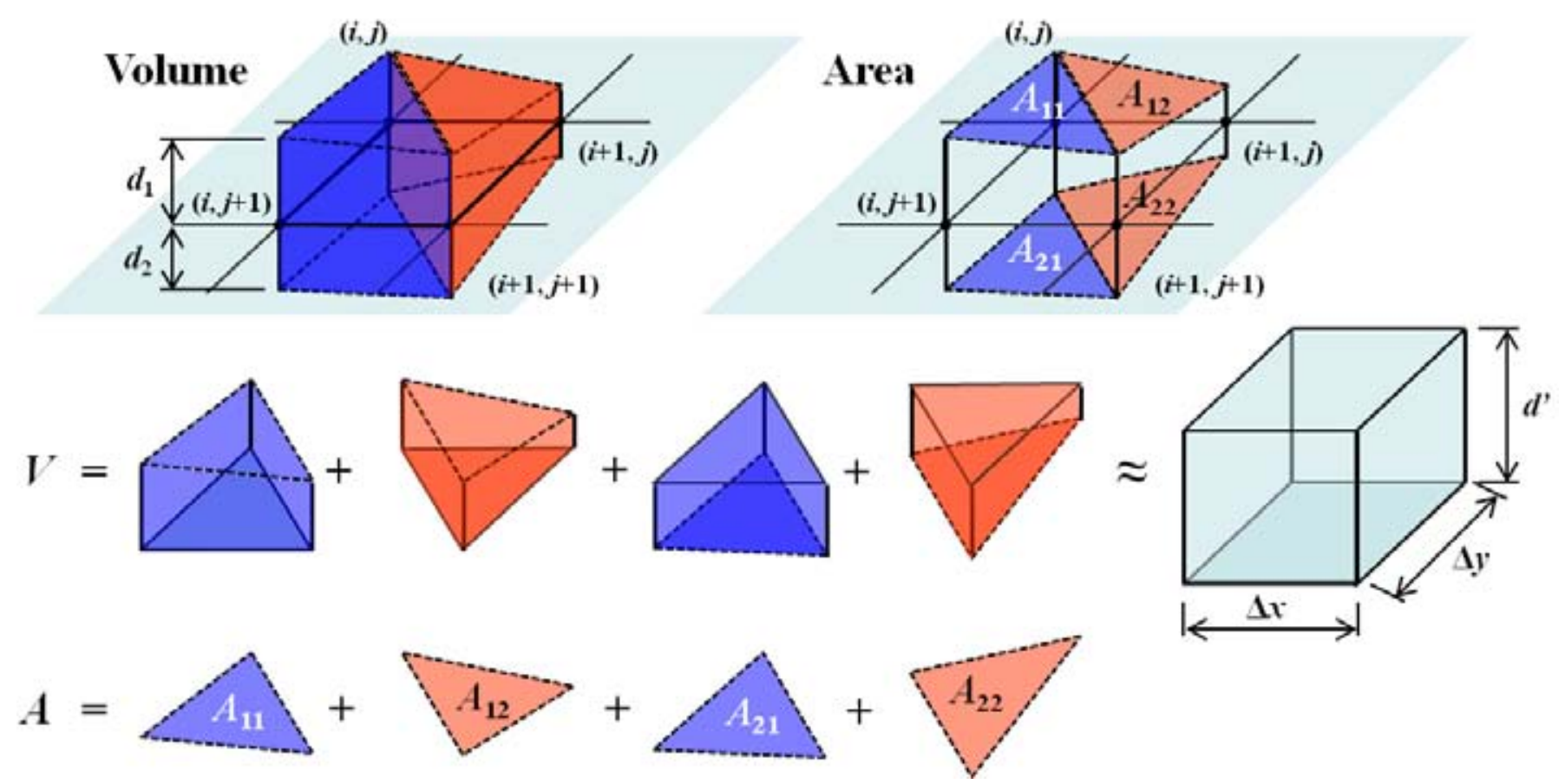

Fig. 9 Estimation of local volume and interfacial area from the thickness of the bubble

$$
\begin{aligned}
& d_{1}=\frac{s}{2}-\delta_{1}, d_{2}=\frac{s}{2}-\delta_{2} \quad\left(\text { for } \alpha_{c}>0.8\right) \\
& d_{1}=d_{2}=\frac{s}{2} \times \alpha_{c} \quad\left(\text { for } \alpha_{c} \leq 0.8\right)
\end{aligned}
$$

where $d_{1}$ and $d_{2}$ are the thicknesses of the gaseous phase in the half gap width. $\delta_{1}$ and $\delta_{2}$ are the liquid film thicknesses of both wall sides. The high void fraction value of $80 \%$ is used as a threshold to evaluate the film behavior between bubbles and the wall. When the void fraction is above the threshold, the bubble thickness is estimated by subtracting the film thickness from the gap width. On the other hand, the thickness is calculated from the void fraction value below the threshold. So the bubble thickness in the narrow gap is defined by

$$
d=d_{1}+d_{2} \text {. }
$$

In this reconstruction, the gaseous phase structure is extracted in the measurement area. The typical images of the interfacial reconstruction are shown in Fig. 8. In the bubbly flow, the three-dimensional bubble shape and its variation can be seen as shown in Fig. 8 (a). The images of Taylor bubbles are shown in Fig. 8 (b) and (c). The frontal and rear shapes of the bubbles can be estimated. In Fig. 8 (d), a coalescence of the bubbles is shown. The liquid film behavior of the bubble coalesced is visualized in the image. It is found that the gaseous phase and the liquid film behaviors can be visualized in the churn flow. The coalescence and breakup occur in Fig. 8 (e), and a spot of the thick film moves downward in Fig. 8 (f). From these results, three-dimensional structures of the flow can be visualized by reconstructing the interfaces.

\subsection{Void fraction and interfacial area concentration}

The local void fraction and interfacial area concentration are estimated from the reconstructed data of the bubble thickness. At first, the thickness of bubbles reconstructed

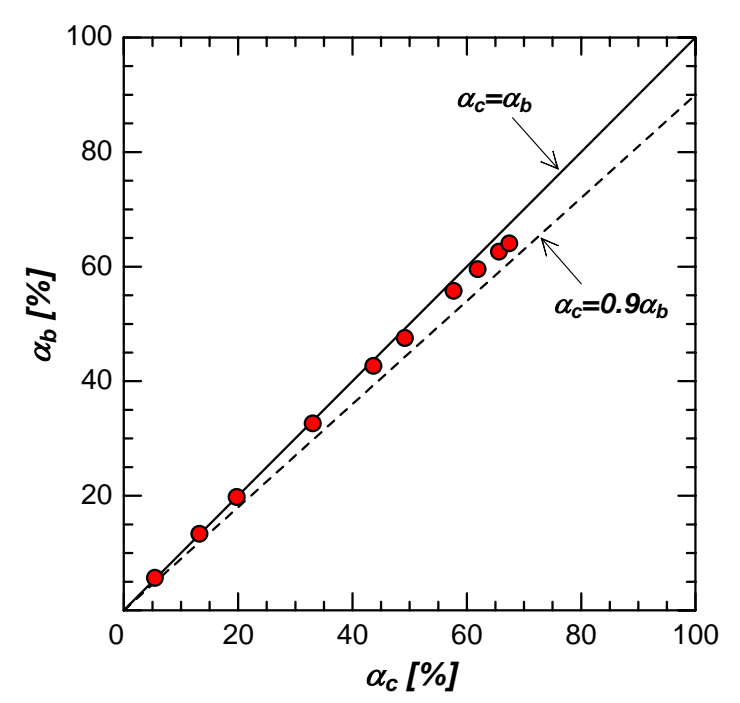

Fig. 10 Comparison of mean void fractions

above is used to make four solids from four measuring points, as shown in Fig. 9. The void fraction is estimated from the volume of the solids and the interfacial area is calculated from the area of the top and bottom face.

To calculate the void fraction, four solids obtained by connecting the points of the gas-liquid interface are approximated for simplicity by a cube with a constant height. So the local void fraction is calculated as follows.

$$
\alpha_{b}=\frac{V}{s \Delta x \Delta y}=\frac{d^{\prime}}{s}
$$

where $d^{\prime}$ is an approximated bubble thickness, $s$ is a gap width, and $\Delta x$ and $\Delta y$ are the horizontal and vertical distances between two measuring points, respectively. Here, the height of the cube in Fig. 9 is calculated by averaging the bubble 


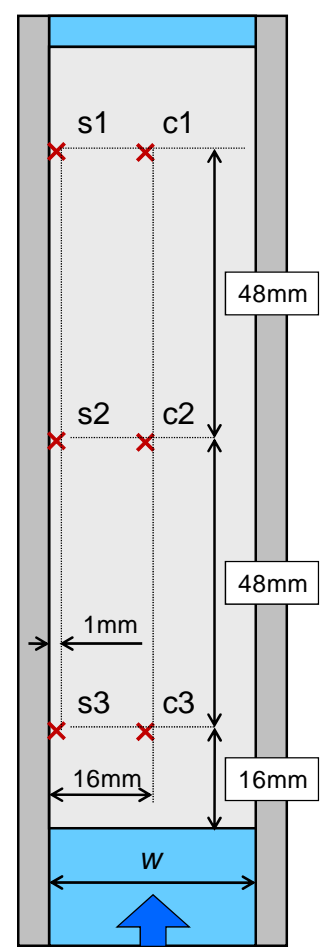

(a) Points

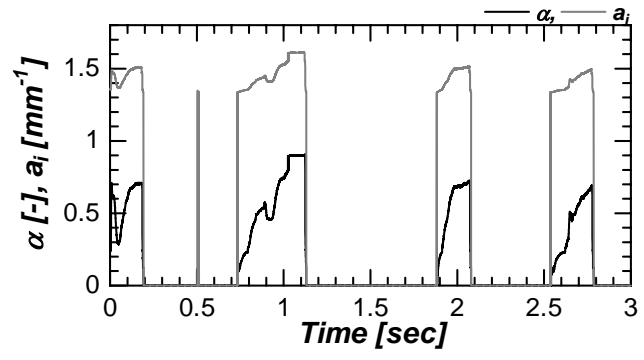

(b) s1

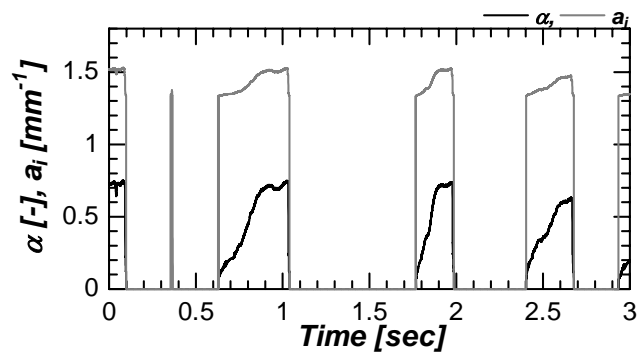

(d) s2

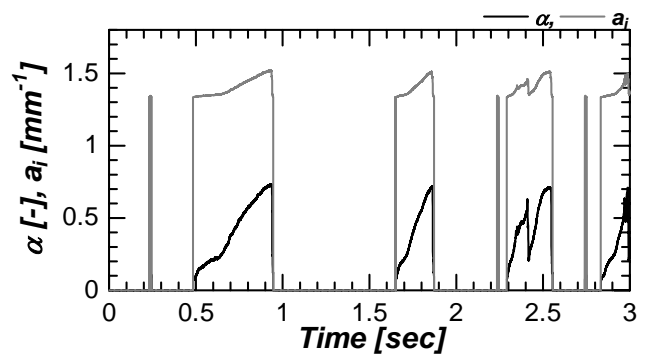

(f) s3

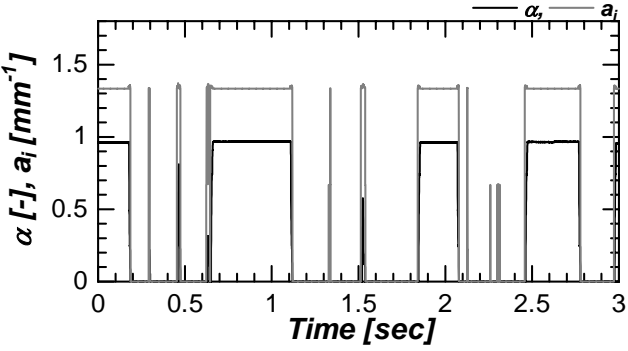

(c) $\mathrm{c} 1$

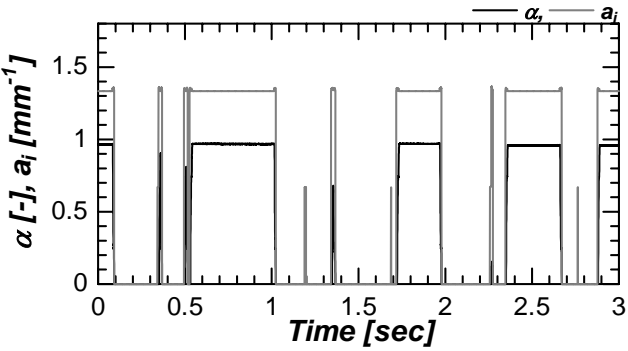

(e) c2

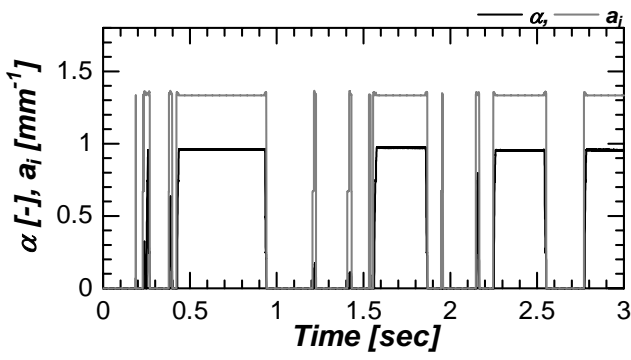

(g) $\mathrm{c} 3$

Fig. 11 Histories of local void fraction and interfacial area concentration in slug flow at $J_{G}=0.15 \mathrm{~m} / \mathrm{s}$

thicknesses at four points, as follows.

$$
d^{\prime}=\frac{d^{(i, j)}+d^{(i+1, j)}+d^{(i, j+1)}+d^{(i+1, j+1)}}{4}
$$

where the bubble thickness $d$ at each measurement point is obtained by Eq. 5. The void fraction recalculated from the bubble thickness is compared with that estimated directly from the electrical conductance. The result of the comparison is shown in Fig. 10.

The void fraction is averaged spatially and temporally. In this figure, the horizontal axis is the void fraction estimated by Eq. 3 and the vertical axis is that estimated by Eq. 6 . The error is small in the lower void fraction condition. However, it increases as increasing the void fraction, and the void fraction from the electrical conductance is overestimated. This is because the void fraction from the conductance does not considered the liquid film thickness between the gaseous phase and the wall of the channel. So the void fraction obtained from the bubble thickness becomes bit lower at high void fraction conditions which have significant liquid film behavior.

The interfacial area is calculated by Heron's formula which estimates the area from the lengths of three sides of a triangle. Four triangles are produced from four bubble thicknesses on each wall side, and the interfacial area formed by four measurement points is a sum of the area of their triangle, as shown in Fig. 9. Then, the local interfacial area concentration is calculated as follow.

$$
a_{i}=\frac{A}{s \Delta x \Delta y}
$$

Thus, the spatio-temporal distributions of the void fraction and interfacial area concentration in the measurement area are obtained by using these estimation methods.

The time-series profiles of the local void fraction and interfacial area concentration in the slug flow are shown in Fig. 11. Fig. 11 (a) represents the point where the local void fraction and interfacial area concentration are estimated for Fig. 11 (b) (g). The points, $\mathrm{c} 1 \sim \mathrm{c} 3$, are located in the middle of the channel width, and $s 1 \sim s 3$ are at $1 \mathrm{~mm}$ from the side wall. From these results, the profiles of the local void fraction and interfacial area concentration show similar tendency at each point. In the profiles of c1, c2 and c3, although the void fraction is almost constant while the bubble passes the point, the interfacial area concentration increases a little at the front and rear of the bubble. This means that the interfacial area becomes larger due to the slope of the bubble edge. In addition, when the slug bubble passes through the measurement point, the void fraction is about $100 \%$ at the center point. However, the void fraction at the side increases with the bubble passage. This shows that the width of the slug bubble grows. Furthermore, comparing between s1(c1), s2(c2) and s3(c3), the time delay is seen in the axial direction. The bubble rising velocity can be estimated from this delay.

Copyright (C) 2011 by JSME 


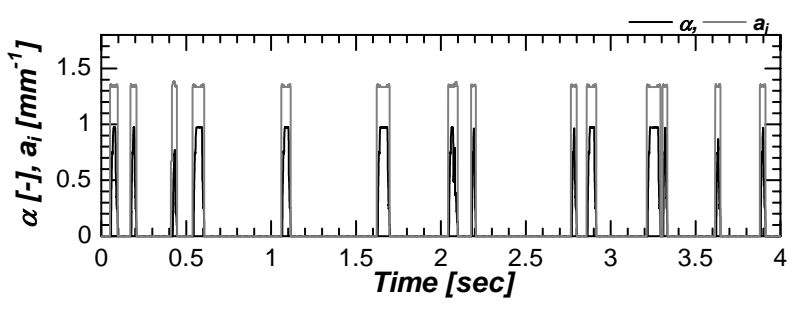

(a) $\mathrm{c} 1$

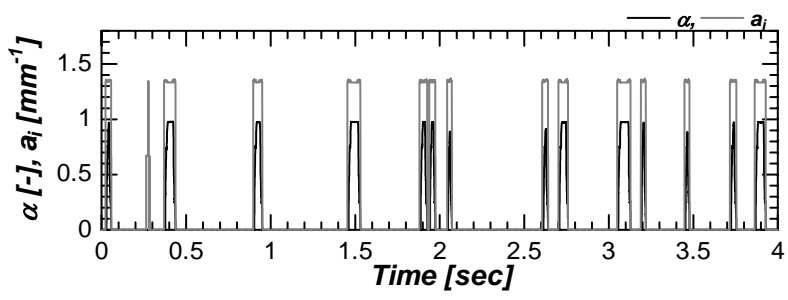

(b) c2

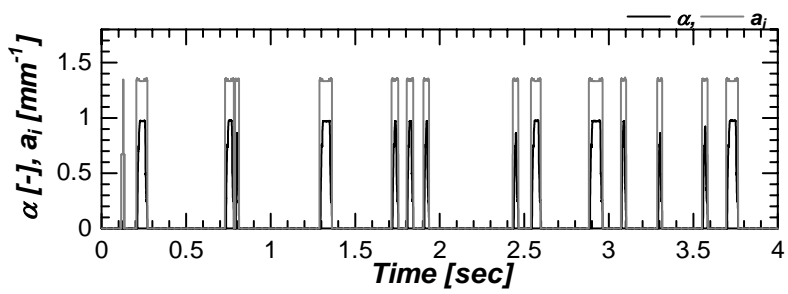

(c) c3

Fig. 12 Histories of local void fraction and interfacial area concentration in bubbly flow at $J_{G}=0.024 \mathrm{~m} / \mathrm{s}$

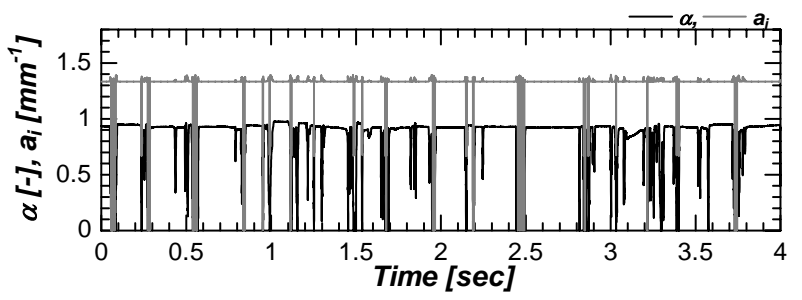

(a) $\mathrm{c} 1$

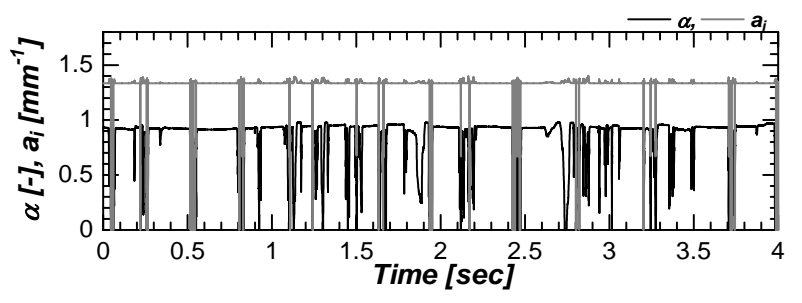

(b) c2

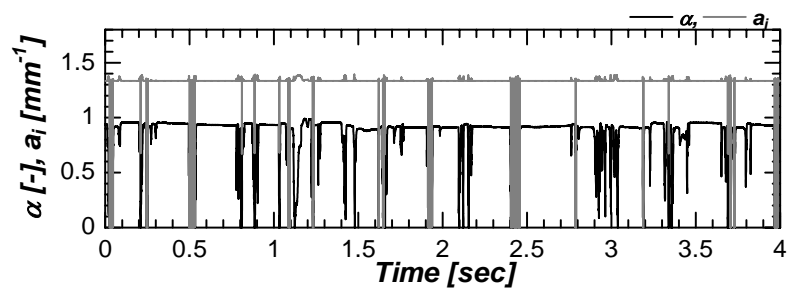

(c) c3

Fig. 13 Histories of local void fraction and interfacial area concentration in churn flow at $J_{G}=1.8 \mathrm{~m} / \mathrm{s}$

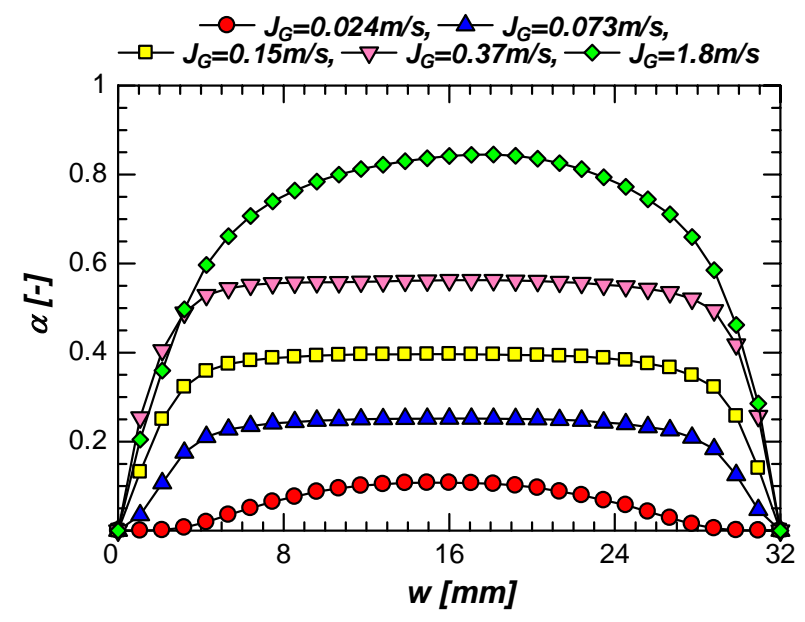

Fig. 14 Mean void fraction profiles along the channel width

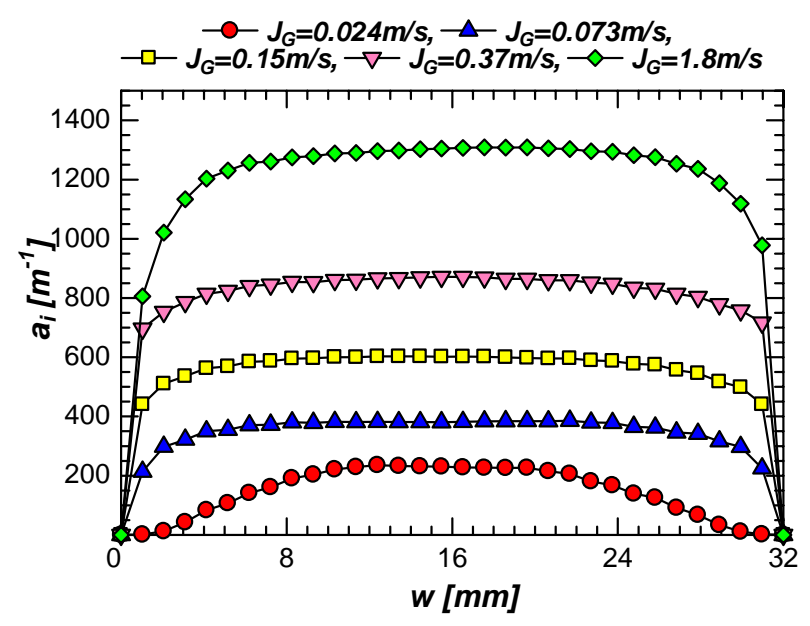

Fig. 15 Mean interfacial area concentration profiles along the channel width

The time-series profiles in bubbly and churn flows are show in Fig. 12 and Fig. 13, respectively. These results are obtained at the centre of the channel width. The small bubbles pass through the measuring point in Fig. 12. In the interfacial area concentration, there is the increase of the measured value at the end of the bubbles, like the Taylor bubbles in Fig.11. In addition, it is found that some bubbles coalesce as the bubbles flow upward. The result of the churn flow has higher void fraction and interfacial area concentration. From these results, the axial movement of the bubbles can be seen in the profiles of the bubbly and slug flows. However, it is difficult to find the relation between these profiles in churn flow.

The mean void fraction profiles along the channel width are shown in Fig. 14. The magnitude increases by a growth of the superficial gas velocity. In bubbly flow at $J_{G}=0.024 \mathrm{~m} / \mathrm{s}$, the profile has a peak at the middle of channel, because most of bubbles flow near the centre. On the other hand, the slug flows $\left(J_{G}=0.073,0.15\right.$ and $0.37 \mathrm{~m} / \mathrm{s}$ ) have flat profiles. This means that there are many Taylor bubbles which have large bubble width. Finally, the profile becomes core peak shape in 
churn flow at $J_{G}=1.8 \mathrm{~m} / \mathrm{s}$. Fig. 15 shows the mean interfacial area concentration profiles. This result shows also the growth of the profile by increasing superficial gas velocity. These profiles are wider than the void fraction profiles in Fig. 14, because the interfacial area is bigger at the bubble edge.

\section{SUMMARY}

The simultaneous measurements of the void fraction and the liquid film thickness were carried out to visualize the gas-liquid interfacial structure in a narrow channel with a gap width of $1.5 \mathrm{~mm}$ by using two liquid film sensors developed by Damsohn and Prasser (2009). The two-dimensional distribution of the liquid film thickness was estimated from the electrical conductance on the sensor and that of the void fraction was obtained by the conductance between the opposing sensors. The gas-liquid interfaces were reconstructed from the measured distributions of the void fraction and the film thickness. These results could show the three-dimensional shape of the bubbles, the bubble coalescence and the liquid film behavior on the gaseous phase. Using the reconstructed data, the void fraction was recalculated and the interfacial area concentration was calculated. The time-series variation and the mean characteristics of both parameters were used to evaluate the interfacial structure in the narrow channel.

\section{REFERENCES}

Damsohn, M. and Prasser, H.-M., 2009, Flow Meas. Instrum., Vol. 20, pp.1-14.

Damsohn, M. and Prasser, H.-M., 2009, Meas. Sci. Technol., Vol.20, 114001.

Damsohn, M. and Prasser, H.-M., 2010, . Nucl. Eng. Design, Vol.240, No. 10, pp. 3126-3144.

Fukaya, Y., et al., 2009, Annals Nucl. Energy, Vol. 36 pp.1374-1381.

Ito, D., et al., 2010, Trans. JSME B Vol.76, No.763, pp.481-482. in Japanese.

Iwamura, T., et al., 2006, Nucl. Eng. Design, Vol. 236, pp.1599-1605.

Kawahara, A., et al., 2008, J. Power Energy Systems, Vol.2, No.1, pp.283-294.

Kureta, M., 2007, J. Power Energy Systems, Vol.1, No.3, pp.211-214.

Kureta, M., 2007, J. Power Energy Systems, Vol.1, No.3, pp.225-238.

Prasser, H.-M., et al., 1998, Flow Meas. Instrum., Vol.9, pp.111-119.

Sadatomi, M., et al., 2007, J. Power Energy Systems, Vol.1, No.2, pp.143-153.

Tamai, H., et al., 2006, J Nucl. Sci. Technol., Vol.43, No.6, pp.699-706.

Uchikawa, S., et al., 2007, J Nucl. Sci. Technol., Vol.44, No.3, pp.277-284. 